\title{
Construction and Implementation of Big Data in Healthcare in Yichang City, Hubei Province
}

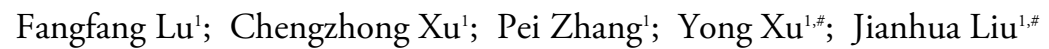

\begin{abstract}
Summary
Environmental pollution, aging, emerging infectious diseases, and unhealthy lifestyles are affecting human health and resulting in serious social and economic burdens. The government-led healthcare big data platform in Yichang has continuously worked towards exploring the use of comprehensive health-related information through top-level design and scientific planning. The platform is based on the following principle: "Openness, inclusiveness, and win-win cooperation." So far, by relying on one-to-one verification, comparison, correlation, and correction with the source, the platform has succeeded in establishing a unique foundation to benefit the people, government, medical care, and health service. Its successes can hopefully act as a positive case for exploring domestic healthcare big data.
\end{abstract}

To solve challenges brought forth by economic development, social transformation, environmental pollution, aging, and poor lifestyles on population health and disease burdens, Healthy China 2030 Plan proposed promoting the application of healthcare and medical big data (1). The Chinese government also lists the application and development of healthcare big data as an important national task (2). The Alibaba Health Strategy Platform (3), Tencent Smart Medical Platform, and regional healthcare big data centers (4) such as Shanghai, Ningbo, Fujian, and Jiangsu have emerged to explore how big data can help solve problems people encounter in their everyday lives.

Lying in the western part of China's Hubei Province, Yichang City is located at the conjunction of the middle and upper reaches of the Yangtze River. Yichang has five districts, three county-level townships, five counties and one national-level high-tech zone. It covers an area of 21,000 square kilometers and has a population of 4.1379 million, including a registered population of 3.9094 million. As one of the first National Pilot City and Healthy City Using Information to Benefit the People, Yichang began to explore the construction of a big data platform from 2014, formed the current platform in 2016, and consistently improved it over the last 3 years.

The government-led Yichang Healthcare Big Data Center adopts the cloud model under an urban framework that breaks barrier of information sharing among different sectors (Figure 1) in China for the first time (5-6). There are over 4 billion pieces of stored data in Yichang Healthcare Big Data Center and more than 1.8 million pieces of data being generated daily covering all 13 counties and districts and the 4.1 million residents. The Yichang regional public health information platform passed the Assessment of Standardization Maturity of National Medical and Health Information Interconnection and achieved the highest grades. After nearly four years of practice and exploration, disease discovery and reporting and health management and delivery has changed dramatically.

Based on one-to-one sources and information sharing, new technologies such as artificial intelligence were used to monitor and report specified infectious diseases, tumors, cardiovascular and cerebrovascular events, hypertension, and diabetes in real time (Figure 2).

Figure 3 shows the in-patient and out-patient admission among total and special populations (children under 5 years old, middle school students, and people older than 65 years old), number of vaccinations, 4 categories of disease event reports (cardiovascular and cerebrovascular diseases, tumors, causes of death, and infectious diseases), daily births and deaths, and other medically related events (for example, first-aid, blood donation and transfusion). It also outlines the follow-up of hypertension and diabetes required by Basic Public Health Services.

Yichang's healthcare big data platform has transformed the city's data collection, integration, sharing, and analysis of population health and healthrelated information, which contains medical and environmental indicators, birth and death registrations, insurance claims, and other important health indicators. Successful information sharing among different departments is meaningful for policymaking 


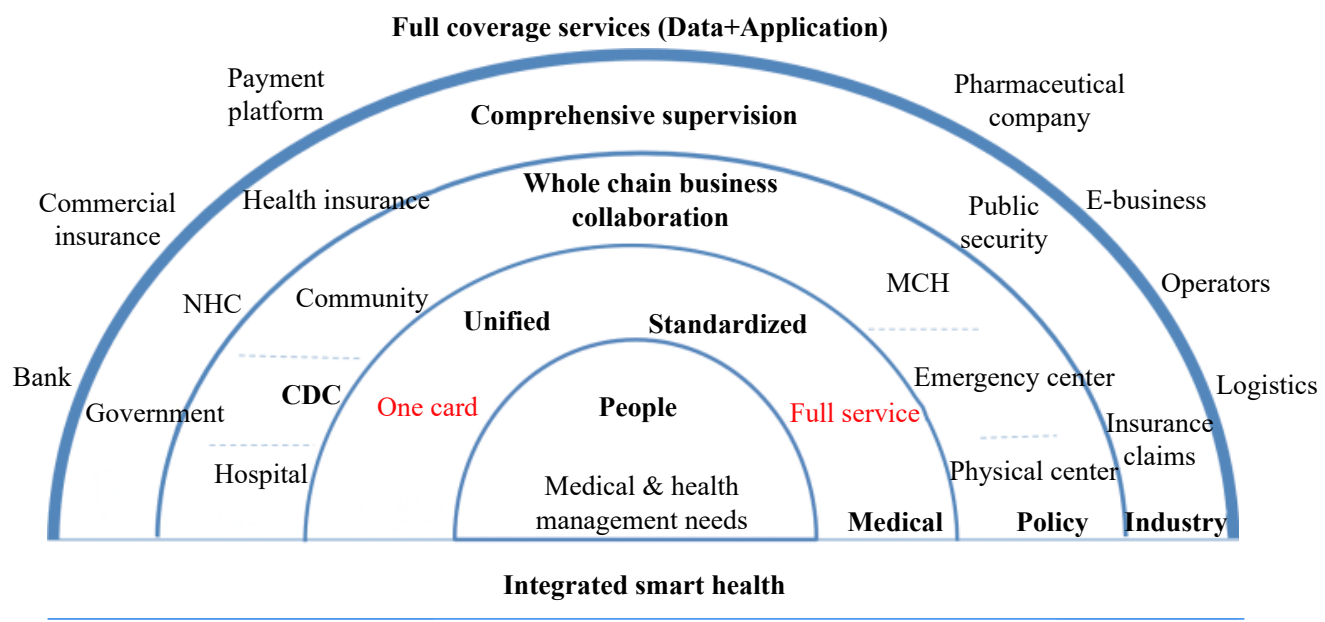

FIGURE 1. The regionally integrated smart healthcare big data platform in Yichang, Hubei province, 2020.
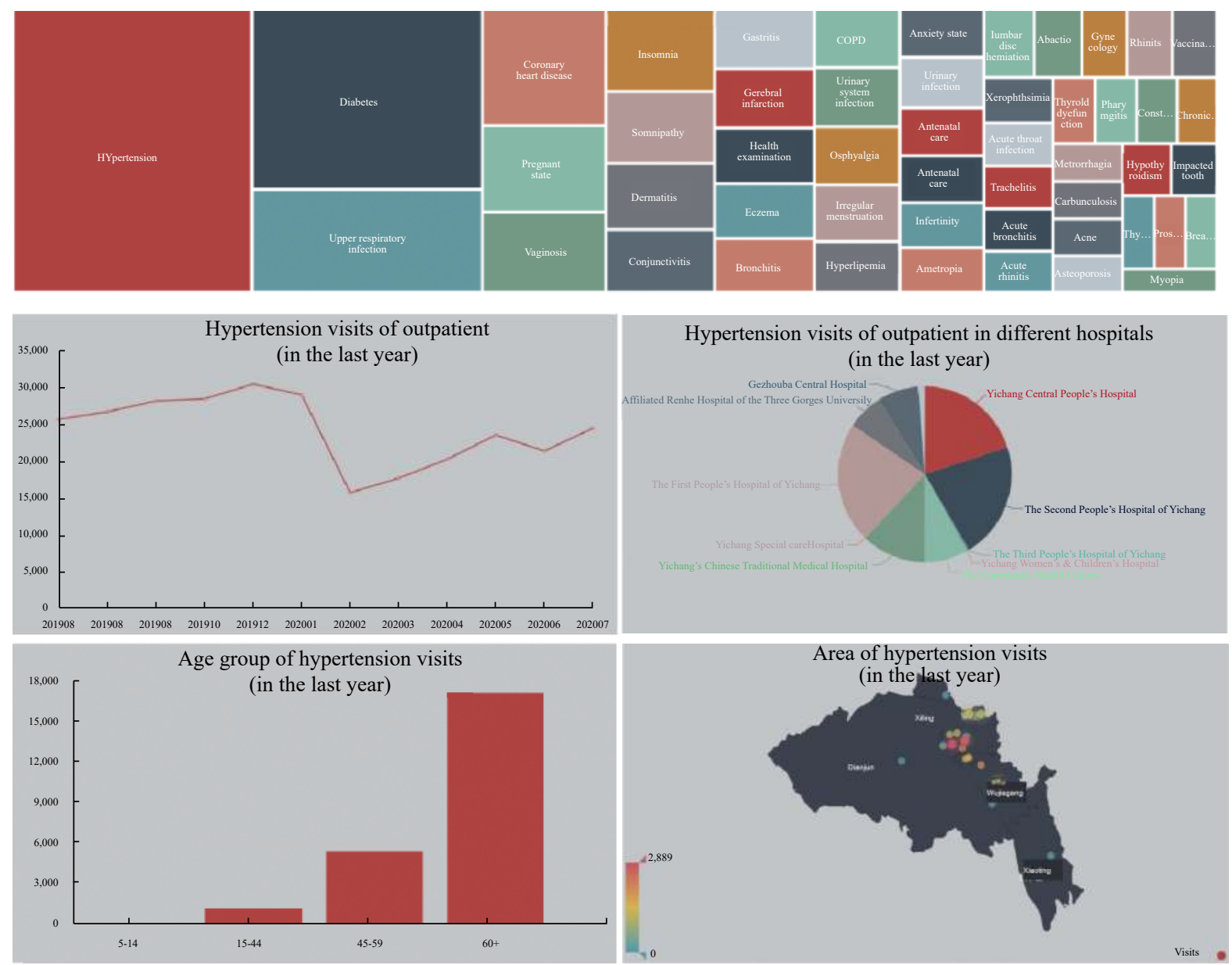

FIGURE 2. The monthly out-patient admissions of Yichang City displayed in real-time on the Healthcare Big Data Platform, Hubei Province, 2020.

and resource allocation. Many innovations have been made, especially in disease monitoring, health management, and "healthy city" construction. First, intelligent discovery and reporting systems of notifiable infectious diseases, hypertension, diabetes, tumors, cardiovascular and cerebrovascular events have been developed. Similar systems can lay a solid foundation for the full coverage of disease monitoring on health 


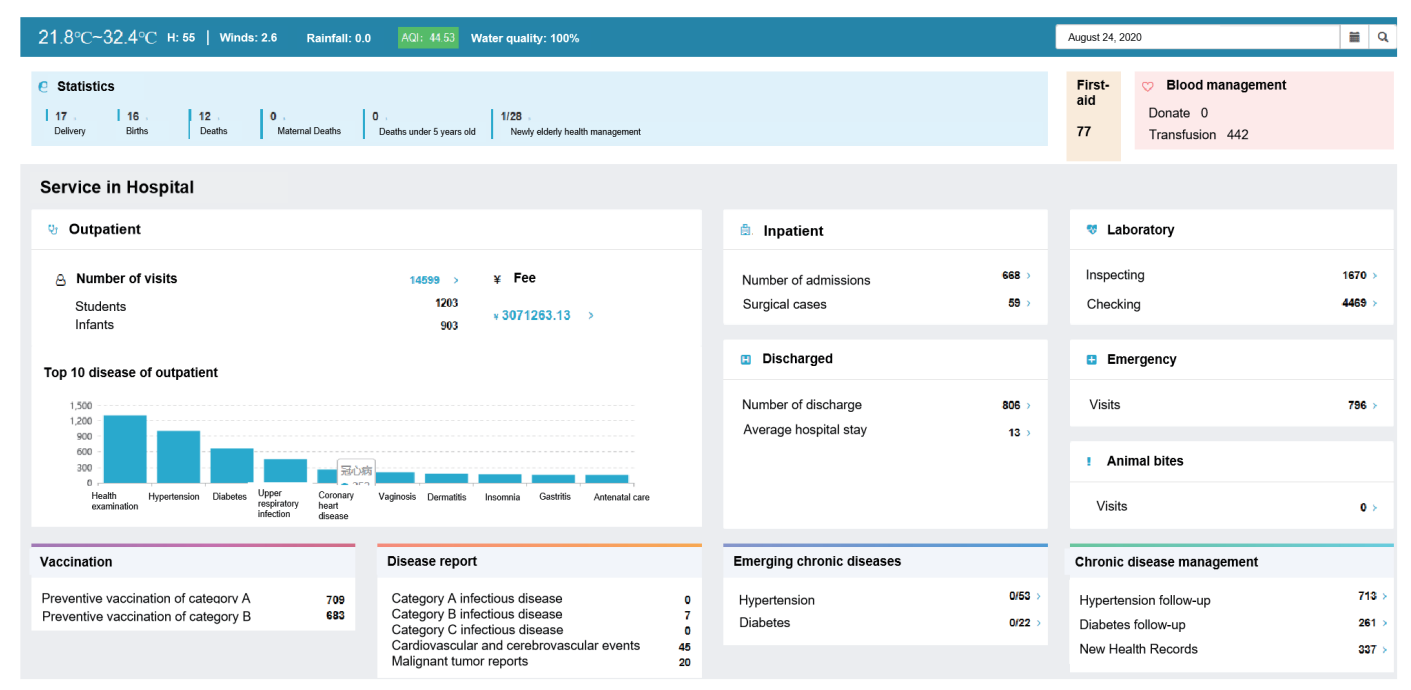

FIGURE 3. Healthcare big data information integration display platform in Yichang City, Hubei Province, 2020.

risk factors, diagnosis, treatment, etc. and allow for deep exploration of disease occurrence and development. Second, combined with personal electronic health records and mobile applications, a full lifecycle and full chain of healthcare information application platform can be built for all permanent residents. Third, integrated department data will also benefit "healthy city" construction by allowing for enhanced urban construction planning and city health evaluation. Functions and advantages of healthcare big data on emergent events of public health such as the coronavirus disease 2019 (COVID-19) pandemic, which emerged in Hubei Province, were also crucial to the response.

One of the most efficient ways to prevent the spread and prevalence of disease is to carry out a detailed and thorough epidemiological investigation to uncover the characteristics of outbreaks. The onset time, symptoms, diagnosis, treatment, laboratory testing, results, underlying diseases of the patient, the departments and doctors involved, and other helpful information can be obtained from the healthcare big data platform to help quickly ascertain the index case, disease characteristics, susceptible populations, and other important evidence. Using big data to assist epidemiological investigations efficiently and accurately has also protected investigators and provided an important basis for government decisions in Yichang.

Another application related to COVID-19 is the Grid Management Information System for joint prevention and control of major epidemics, which was developed by integrating the information data of multiple channels such as the national temperature detection platform, short message reporting platform, and the grid agent reporting system to collect information including dynamic temperature data and information of key prevention and control personnel including those returning or coming to Yichang, members of these families, close contacts, and pyrexia patients. Intelligent prevention and control programs were set up for different personnel and automatically distributed to each township and urban neighborhood office for isolation management and medical observation. The dynamic supervision applied in urban areas in Yichang has avoided heavy and complicated reports, provided strong evidence for emergency management and early warnings, and allowed for closed-loop management of all personnel.

By using the aspects of the big data platform for patient data, improvements in timeliness, accuracy, and integrity of data can be tracked and displayed. Missing, delayed, and repeated reports can be prevented. The medical records of patients who were diagnosed as COVID-19 in the big data platform were also useful for doctors to make more informed treatment plans.

In the big data era, accurate, comprehensive, and available data will be the source of high-quality developments of healthcare in the future and the basis and key to decision making ( 7 ). To construct a "smart and healthy city," Yichang's Healthcare Big Data Center has carried out some beneficial practices and explorations in data standardization, interconnection, and sharing. However, further effort should be taken to guarantee long-term, stable, high-quality data sources. In addition, deeper and broader explorations 
are needed to use big data for monitoring diseases and health, allocating public resources, supporting government decisions, and serving people's health.

doi: $10.46234 / \mathrm{ccdcw} 2020.254$

\# Corresponding authors: Yong Xu, hbycxy2007@163.com; Jianhua Liu, amour_1114@163.com.

${ }^{1}$ Yichang Center for Disease Control and Prevention,Yichang, Hubei, China.

Submitted: August 28, 2020; Accepted: November 12, 2020

\section{REFERENCES}

1. Central Committee of Chinese Communist Party. State Council. The Plan for Healthy China 2030. Beijing: Government of the People's Republic of China, 2016. http://www.gov.cn/xinwen/2016-10/25/ content_5124174.htm. [2020-11-11]. (In Chinese).

2. General Office of the State Council, Guiding opinions of the general office of the State Council on promoting and standardizing the application and development of health and medical big data. Beijing: Government of the People's Republic of China, 2016. http://www.gov. cn/zhengce/content/2016-06/24/content_5085091.htm. [2020-11-11]. (In Chinese).

3. Huang YT. Full analysis of Alibaba health platform strategy. HighTechnol Ind 2017;(4): 52-6. http://www.wanfangdata.com.cn/details/ detail.do?_type=perio\&idgkjycyh201704010. (In Chinese).

4. Zhang LX, Wang HB, Li QZ, Zhao MH, Zhan QM. Big data and medical research in China. BMJ 2018;36:55910. http://dx.doi.org/ 10.1136/bmj.j5910.

5. Chen GJ. Breaking the information island to release the dividend of medical big data. Cap Med 2017(5):38 - 9. http://dx.doi.org/ 10.3969/j.issn.1005-8257.2017.05.016. (In Chinese).

6 . Wang J. From e-government, smart city to smart society--analysis on the practice of smart Yichang integration. E-Government 2018(5):52 - 63. http://dx.doi.org/10.16582/j.cnki.dzzw.2018.05.006. (In Chinese).

7. Baillie J. How 'Big data' will drive future innovation. Health Estate 2016;70(3): 59-64. https://pubmed.ncbi.nlm.nih.gov/27132307/. 